\title{
Probing Adsorption Interactions in Metal-Organic Frameworks using X-ray Spectroscopy
}

\author{
Walter S. Drisdell, ${ }^{\dagger}$ Roberta Poloni, ${ }^{\dagger, \ddagger, \S, \|}$ Thomas M. McDonald, ${ }^{\dagger, \S}$ Jeffrey R. Long, ${ }^{\dagger, \S}$ Berend Smit, $^{\dagger, \S}$ \\ Jeffrey B. Neaton, ${ }^{\dagger}$ David Prendergast, ${ }^{\dagger}$ and Jeffrey B. Kortright ${ }^{*}{ }^{\dagger}$ \\ ${ }^{\dagger}$ Materials Sciences Division, Lawrence Berkeley National Laboratory, Berkeley, California 94720, United States \\ ${ }^{\ddagger}$ Department of Chemical and Biomolecular Engineering, University of California, Berkeley, California 94720-1460, United States \\ ${ }^{\S}$ Department of Chemistry, University of California, Berkeley, California 94720-1460, United States \\ "Laboratoire de Science et Ingénierie des Matériaux et Procédés (SIMaP), UMR CNRS 5266, Grenoble-INP, BP 75, 38402 Saint \\ Martin d'Hères Cedex, France
}

\section{Supporting Information}

ABSTRACT: We explore the local electronic signatures of molecular adsorption at coordinatively unsaturated binding sites in the metal-organic framework Mg-MOF-74 using X-ray spectroscopy and first-principles calculations. In situ measurements at the $\mathrm{Mg}$ K-edge reveal distinct pre-edge absorption features associated with the unique, open coordination of the $\mathrm{Mg}$ sites which are suppressed upon adsorption of $\mathrm{CO}_{2}$ and $N, N^{\prime}$ dimethylformamide. Density functional theory shows that these spectral changes arise from modifications of local symmetry around the $\mathrm{Mg}$ sites upon gas uptake and are strongly dependent

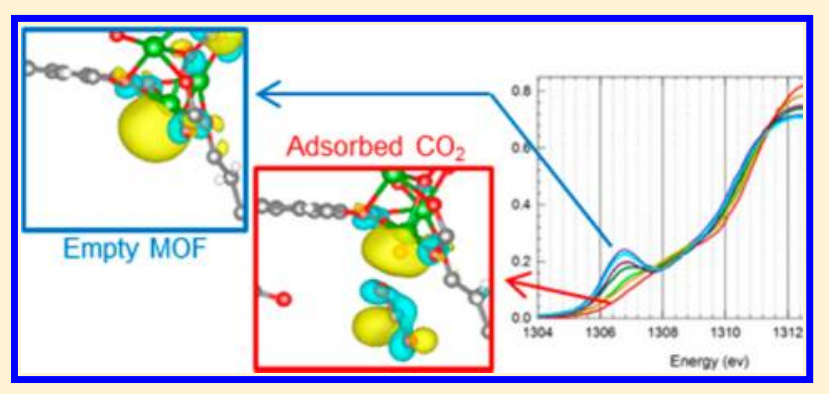
on the metal-adsorbate binding strength. The expanded $\mathrm{MOF} \mathrm{Mg}_{2}(\mathrm{dobpdc})$ displays the same behavior upon adsorption of $\mathrm{CO}_{2}$ and $N, N^{\prime}$-dimethylethylenediamine. Similar sensitivity to local symmetry is expected for any open metal site, making X-ray spectroscopy an ideal tool for examining adsorption in such MOFs. Qualitative agreement between ambient-temperature experimental and $0 \mathrm{~K}$ theoretical spectra is good, with minor discrepancies thought to result from framework vibrational motion.

\section{INTRODUCTION}

Metal-organic frameworks (MOFs) that feature internal pore surfaces replete with open metal sites-five-coordinate metal cations known to behave as Lewis acids - are presently among the most promising solid adsorbents for selectively removing $\mathrm{CO}_{2}$ from dry gas mixtures. Open metal sites preferentially adsorb $\mathrm{CO}_{2}$ over $\mathrm{N}_{2}$ or $\mathrm{CH}_{4}$ due to its larger quadrupole moment and greater polarizability. The moderate heat of adsorption of $\mathrm{CO}_{2}$ onto such sites produces selective, highcapacity adsorbents that can be easily regenerated via temperature or vacuum swing methods. The MOF-74 series of MOFs have been widely studied for their high performance in both gas separation and hydrogen storage applications. ${ }^{1-5}$ In particular, Mg-MOF-74 (CPO-27-Mg, $\mathrm{Mg}_{2}$ (dobdc) (dobdc ${ }^{4-}=$ 2,5-dioxido-1,4-benzenedicarboxylate)) is a promising adsorbent for selective $\mathrm{CO}_{2}$ adsorption due to its large $\mathrm{CO}_{2}$ capacity and high selectivity for $\mathrm{CO}_{2}$ over other gases. ${ }^{6-9}$ This MOF features a unique five-coordinate square-pyramidal geometry around the $\mathrm{Mg}$ sites; unlike other square-pyramidal $\mathrm{Mg}$ complexes, ${ }^{10,11}$ the organic framework in Mg-MOF-74 constrains the bond angles at the $\mathrm{Mg}$ sites and ensures that a significant portion of the $\mathrm{Mg}$ coordination sphere is available for adsorbing other species. The $\mathrm{Mg}$ sites can therefore be considered quasi-octahedral, with the apical site vacant. To date, characterization efforts aimed at a microscopic under- standing of interactions between gases and the adsorbent have consisted primarily of low-temperature $\mathrm{X}$-ray and neutron diffraction experiments, ${ }^{12-14}$ NMR spectroscopy, ${ }^{15}$ infrared spectroscopy, ${ }^{7}$ inelastic neutron scattering, ${ }^{16}$ and theoretical studies. ${ }^{7,17,18}$ These studies have determined a tilted, end-on binding configuration for $\mathrm{CO}_{2}$, but insight into any changes to the electronic structure of the MOF upon $\mathrm{CO}_{2}$ adsorption have so far come solely from theoretical calculations. Experimental examination of these changes would validate theoretical predictions and provide a fundamental understanding of the binding mechanisms in this MOF.

Herein we directly observe changes in specific molecular orbitals at the $\mathrm{Mg}$ site in Mg-MOF-74 upon binding of $\mathrm{CO}_{2}$ and $N, N^{\prime}$-dimethylformamide (DMF) using near-edge X-ray absorption fine structure (NEXAFS) spectroscopy. We also examine such effects in $\mathrm{Mg}_{2}$ (dobpdc) (dobpdc ${ }^{4-}=4,4^{\prime}$ dioxido-3,3'-biphenyldicarboxylate), an expanded analogue of Mg-MOF-74. ${ }^{19}$ These measurements allow us to determine how the electronic interactions differ for different adsorbed species and for binding sites in a larger framework. The NEXAFS technique is element-specific, probing the unoccupied electronic states associated with the excited atom. It provides

Received: September 3, 2013

Published: November 13, 2013 
local electronic structure and coordination information due to the overlap between the core-level state and the empty final state and, for the soft X-rays used in this study, adherence to optical dipole selection rules. For systems with specific, chemically distinct binding sites such as the $\mathrm{Mg}$ sites in $\mathrm{Mg}$ MOF-74, NEXAFS spectra provide high sensitivity to changes in the local electronic structure and coordination at the binding sites upon adsorption of gas molecules. A specially designed gas cell allows spectra to be collected under vacuum or under pressures of $\mathrm{CO}_{2}$ as high as 100 Torr at ambient temperature, providing a direct comparison between the empty and bound $\mathrm{Mg}$ sites of the same MOF sample. In this study we demonstrate a strong spectral response to the unique geometry at the open metal sites in both MOFs, revealing a molecular orbital structure that is highly sensitive to adsorbed species and the strength of the interaction through its effects on local symmetry around the metal sites.

We employ plane-wave density functional theory (DFT) within the generalized gradient approximation to calculate the NEXAFS spectrum from optimized DFT structures. This approach has been used previously to calculate NEXAFS spectra for a variety of condensed-phase systems. ${ }^{20-24}$ The DFT calculations not only provide spectra for comparison with experiment but also provide reasonable approximations to the X-ray excited electronic states, indicating how these are modified in the presence of adsorbed species. By coupling experiment and theory in this way, we are able to interpret observed spectral changes through a detailed study of the local electronic structure around the $\mathrm{Mg}$ atom. While photoemission studies have examined the electronic structure of gases adsorbed at metal surfaces, ${ }^{25}$ our study is, to our knowledge, the first in situ observation of the electronic structure changes associated with gas adsorption in MOF interfaces.

\section{EXPERIMENTAL SECTION}

1. Experimental Details. 1.1. Gas Cell Design. The gas cell design is similar to that of an ion chamber, adapted for transmission detection. The cell consists of a small cubic vacuum chamber with a specially designed flange and clamp to mount a silicon nitride membrane in the center. This membrane acts as an X-ray transmissive barrier separating any gas in the cell from the vacuum of the beamline $\mathrm{X}$-ray source, as well as the substrate upon which the transmission samples are mounted (see below). A gas inlet with a valve allows introduction of $\mathrm{CO}_{2}$ gas into the cell. There are two pumps, attached to the cell via a gate valve: a turbomolecular pumping station with dry vacuum pumps suitable for vacuum measurements and a dry scroll roughing pump suitable for measurements with gas present. A second valve allows the membrane to be bypassed when pulling vacuum so it will not burst from the pressure differential. The transmitted X-rays are detected with a GaAsP photodiode, mounted on a linear manipulator to allow the X-ray path length in the cell to be varied from 1 in. down to a few millimeters. Long path lengths are useful for gas-phase measurements, whereas it is advantageous to minimize the path length for measurements of the MOF materials to limit interference from free $\mathrm{CO}_{2}$ gas. The housing of the photodiode was removed to allow X-ray detection. The entire cell can be sealed to ensure that activated MOF samples (i.e., samples in which all solvent is removed from the pores) are not exposed to water vapor when transporting the cell to a beamline for measurements.

1.2. Sample Preparation. The full details for the synthesis and preparation of samples are given in the Supporting Information. In brief, the synthesized MOF powder was suspended in a small amount (ca. $0.5 \mathrm{~mL}$ ) of solvent (acetone, $\mathrm{MeOH}$, or hexanes). The suspension was then deposited onto a $\mathrm{Si}_{3} \mathrm{~N}_{4}$ membrane, and the solvent was allowed to evaporate, leaving a thin coating of powder on the membrane. For measurements of activated MOFs, the entire membrane with powder coating was loaded into a glass evacuation chamber and heated under vacuum to activate the MOF powder (see the Supporting Information for details on the activation procedure). Activated samples were then stored under dry nitrogen and loaded into the gas cell in a dry nitrogen glovebox to avoid exposure to water vapor before measurement.

1.3. NEXAFS Spectra. NEXAFS spectra were collected at bending magnet beamline 6.3.1 ( $10^{11}$ photons/s) at the Advanced Light Source at Lawrence Berkeley National Laboratory. The gas cell was mounted on an XY manipulator and attached to the downstream port on the beamline. Due to imperfect control of the powder sample thickness, care was taken to ensure that spectra could be meaningfully compared to one another. For a given sample, initial alignment using the $\mathrm{Mg} \mathrm{K}$ edge absorption was performed under vacuum. Once aligned, the sample was not moved again, so that all spectra were collected on the same spot on the sample with the same thickness and morphology. The first Mg K-edge NEXAFS spectrum of each sample was collected under vacuum, followed by progressively higher pressures of $\mathrm{CO}_{2}$ gas. Finally, the gas was pumped out and the spectrum collected under vacuum again, to determine if any observed spectral changes were reversed.

To ensure proper normalization of the measured spectra, spectra of the incident X-ray beam were also collected using a blank silicon nitride membrane of $100 \mathrm{~nm}$ thickness under vacuum. To get the final spectra, we used the relation

$$
\frac{I}{I_{0}}=e^{-\mu t}
$$

where $I$ is the intensity of the transmission spectrum, $I_{0}$ is the intensity of the direct beam spectrum, $\mu$ is the absorptivity, and $t$ is the thickness of the sample. By solving for $\mu t$, we can estimate the apparent thickness of the sample under the assumption that it is uniformly thick. Because these powder samples are not uniformly thick, absolute intensities of different spectral features may be distorted relative to each other; therefore, we do not interpret absolute intensities in this study. Instead, we emphasize reversible spectral changes that occur when $\mathrm{CO}_{2}$ gas is present and note that any distortions in absolute spectral intensities do not change during the measurement, as the same sample volume is always measured. Any spectral changes observed with this method must therefore arise from interactions with $\mathrm{CO}_{2}$ gas and not from sample morphology. To generate comparable spectra for a given sample, we started with $\mu t$ as a function of photon energy and subjected these spectra to a linear pre-edge baseline correction and intensity normalization necessary to account for changing gas absorption with pressure. After normalization, we rescaled the intensity to match the post-edge $(1374 \mathrm{eV})$ step height observed in the baseline-corrected $\mu t$ spectrum under vacuum. The intensity scale therefore provides an estimate of the apparent optical density of the sample due to the $\mathrm{Mg}$ sites only, without any contributions from $\mathrm{CO}_{2}$ gas. These spectra were then compared to identify any spectral changes at the $\mathrm{Mg}$ sites signifying the binding of $\mathrm{CO}_{2}$ in the materials. The photon energy was calibrated by collecting a total electron yield (TEY) spectrum of $\mathrm{MgO}$, shown in Figure S2 (Supporting Information), and aligning the sharp low-energy spectral feature with that measured previously by Lindner et al. ${ }^{26}$

2. DFT Calculations. 2.1. Structural Relaxation. The structural relaxation of Mg-MOF-74 and $\mathrm{Mg}_{2}$ (dobpdc) for the bare frameworks and for the frameworks in the presence of adsorbed molecules $\left(\mathrm{CO}_{2}\right.$ dimethylformamide, and $N, N^{\prime}$-dimethylethylenediamine) are performed using the SIESTA package. ${ }^{27,28}$ The vdW-DF2 functional is employed to account for long-range dispersion forces. ${ }^{29} \mathrm{~A}$ recent work $^{18}$ shows that this approach can correctly predict the experimental heat of adsorption of $\mathrm{CO}_{2}$ in MOFs. Norm-conserving TrouillerMartin pseudopotentials ${ }^{30}$ are used in these calculations. $2 \mathrm{~s}$ and $2 \mathrm{p}$ electrons of $\mathrm{C}, \mathrm{N}$, and $\mathrm{O}$ atoms are explicitly included in the valence; for $\mathrm{Mg}$, semicore electrons are considered (2s, $2 \mathrm{p}$, and $3 \mathrm{~s})$. We use a variationally optimized ${ }^{31}$ double- $\zeta$ polarized basis set including $d$ orbitals for all atoms. The integration over the Brillouin zone is carried over $64 k$-points. ${ }^{32}$ Real space integrals are performed on a mesh with 
a 280 Ry cutoff. For each calculation, we optimize the atomic positions of all atoms until forces are smaller than $40 \mathrm{meV} / \AA$. The relaxed structures are then used to compute NEXAFS spectra. The binding energy between the MOF at the Mg site and the different adsorbed species is computed using vdW-DF2. The $\mathrm{CO}_{2}$ and DMF binding energies in Mg-MOF-74 are 41.3 and $108.1 \mathrm{~kJ} / \mathrm{mol}$, respectively. The $\mathrm{Mg}-\mathrm{O}$ bond length is $2.33 \AA$ for $\mathrm{CO}_{2}$ and $2.07 \AA$ for DMF. For $\mathrm{Mg}_{2}\left(\right.$ dobpdc), the $\mathrm{CO}_{2}$ and mmen binding energies are 39.3 and 105.8 $\mathrm{kJ} / \mathrm{mol}$, respectively, with $2.40 \AA$ and $2.37 \AA \mathrm{Mg}-\mathrm{O}$ bond lengths.

2.2. Calculated NEXAFS Spectra. Starting from the relaxed ground state structures computed using the methodology discussed in the previous section, we simulate X-ray absorption spectra (XAS) within a plane-wave pseudopotential orbital-occupancy-constrained DFT framework, employing the PBE functional. ${ }^{33}$ We expect this functional to be adequate for computing the spectra, since the employed geometries, computed using the vdW-DF2 functional, are in good agreement with experiments. ${ }^{18,34}$ The transition amplitudes are calculated according to Fermi's golden rule expression for the X-ray absorption cross-section: ${ }^{35}$

$$
\sigma(\omega)=4 \pi^{2} \alpha_{0} \hbar \omega \sum_{\mathrm{f}}\left|M_{\mathrm{i} \rightarrow \mathrm{f}}\right|^{2} \delta\left(E_{\mathrm{f}}-E_{\mathrm{i}}-\hbar \omega\right)
$$

Here $\alpha_{0}$ is the fine structure constant, $\hbar \omega$ is the energy of the absorbed photon, and $M_{\mathrm{i} \rightarrow \mathrm{f}}$ are the transition amplitudes between the initial (i) and final (f) states, with corresponding energy difference $E_{\mathrm{f}}-E_{\mathrm{i}}$. Within the electric-dipole approximation, we further approximate the transition amplitudes as effective single-particle matrix elements:

$$
M_{\mathrm{i} \rightarrow \mathrm{f}}=\left\langle\Psi_{\mathrm{f}}|\hat{\varepsilon} \cdot \mathbf{R}| \Psi_{\mathrm{i}}\right\rangle \approx S\left\langle\psi_{\mathrm{f}}|\hat{\varepsilon} \cdot \mathbf{r}| \psi_{\mathrm{i}}\right\rangle
$$

where $\hat{\varepsilon}$ is the polarization direction of the electromagnetic vector potential, $R$ and $r$ are the many-electron and single-electron position operators, and $\Psi_{\mathrm{i}, \mathrm{f}}$ are the many-body initial and final states. The effective single-particle states, $\psi_{\mathrm{i}, \mathrm{f}}$, are Kohn-Sham eigenstates of the ground and excited state self-consistent fields, respectively. In this wok, the initial state is always the $1 \mathrm{~s}$ orbital of the $\mathrm{Mg}$ atom and the final states are accessible (i.e., unoccupied) Kohn-Sham eigenstates derived from a self-consistent field computed within the excited electron and core-hole $(\mathrm{XCH})$ approximation for the electronic final state. ${ }^{36}$ In this final-state-rule approach, the excited-state electron density is computed self-consistently subject to two constraints: (1) replacement of the ground-state pseudopotential of the core-excited atom in the given system with that derived from a similarly core-excited isolated atom (for the Mg K-edge we would assume an electronic configuration of $\left.1 s^{1} 2 s^{2} 2 p^{6} 3 s^{2} 3 p^{1}\right)$ to model the hole and (2) the addition of one extra (excited) electron to the total number of ground-state valence electrons of this system.

Calculated spectra are computed from eq 2 with the $\delta$ function replaced by a Gaussian convolution with finite width (typically $0.3 \mathrm{eV}$ ). We did not make estimates of the core-hole lifetime of the $\mathrm{Mg} 1 \mathrm{~s}$ coreexcited state, which would impart a significant Lorentzian broadening to each computed transition. The sum over final states in our supercell calculations includes both electronic band indices and wave vectors (so-called $k$-point sampling of the first Brillouin zone). The spectral line shape is derived from a numerically converged sampling of eigenenergies and transition matrix elements within the first Brillouin zone. Typically this can require several $k$-points, and we reduce the computational effort of such calculations by exploiting a recently developed implementation of the Shirley interpolation scheme, ${ }^{37}$ from which the entire Brillouin zone can be generated based only on information from the zone center $(k=0)$. In order to determine the band indices of dominant transitions in calculated spectra before convolution and neglecting $k$-point sampling, in some cases we show transition probabilities, $\left|M_{\mathrm{i} \rightarrow \rightarrow}\right|^{2}$, from the Brillouin zone center only. Similarly, to interpret such transitions, we also examine isosurfaces of the electronic component of the excited state, approximated as a Kohn-Sham eigenstate of the XCH self-consistent field at $k=0$.

In this work, we compare $\mathrm{Mg} \mathrm{K}$-edge spectra for various MOFs with and without absorbed species or functionalization. Our approach uses pseudopotentials for efficient plane-wave representation of the valence electronic structure only. In the spirit of our $\triangle S C F$ approach, we might compute relative core-excitation energies of $\mathrm{Mg}$ atoms in different systems using total energy differences. However, for systems with differing periodic boundary conditions, total energies are not comparable. Instead, one should compare energies that are independent of system size, and the energy of a charge-neutral atomic excitation embedded in a condensed phase should converge, beyond a certain minimum supercell volume, to a quantity independent of the chosen periodic boundary condition. However, our use of pseudopotentials prevents us from computing meaningful energy differences between excited and ground states, since the addition of a modified pseudopotential to the excited state system adds an unknown shift to the total energy. Instead, we make comparison to a theoretical standard-the isolated excited- and ground-state atom under the same periodic boundary conditions. ${ }^{23}$ This is equivalent to computing a difference in formation energies between the excited and ground states, and formation energies are numerically well-defined within a pseudopotential framework. ${ }^{22}$ In practice, we then align the computed spectrum of a well-defined system ( $\mathrm{MgO}$ in this case) to experiment and use the same constant of alignment in all further calculations using the same excited-state pseudopotential for $\mathrm{Mg}$.

To analyze and interpret our simulated XAS, we imagine the K-edge excitonic excited states to be factorizable into a product of the 1s hole state and the corresponding electronic states of energy greater than or equal to that of the highest occupied state of the $\mathrm{XCH}$ self-consistent field. We plot representations of these electronic states as isosurfaces in $3 \mathrm{D}$ space and explore their symmetry and hybridization in the vicinity of the excited $\mathrm{Mg}$ atom, in order to correlate specific spectral changes with specific adsorption interactions.

\section{RESULTS AND DISCUSSION}

1. NEXAFS Spectra. NEXAFS spectra at the Mg K-edge were collected for Mg-MOF-74 in its activated form and for the same sample in contact with $\mathrm{CO}_{2}$ gas at various pressures. The spectrum of DMF-ligated Mg-MOF-74 (DMF $=N, N^{\prime}$ dimethylformamide) was also collected to explore the spectral dependence on the different strengths of adsorption interactions for different adsorbed species at the $\mathrm{Mg}$ site. DMF is used as a solvent during synthesis of the MOF and interacts strongly with the $\mathrm{Mg}$ site, whereas the $\mathrm{Mg}-\mathrm{CO}_{2}$ interaction is weaker. We also performed $\mathrm{CO}_{2}$ adsorption measurements for $\mathrm{Mg}_{2}$ (dobpdc) ( dobpdc $^{4-}=4,4^{\prime}$-dioxido-3,3'biphenyldicarboxylate), ${ }^{19}$ an expanded analogue to Mg-MOF74 , to explore possible differences in the adsorption interactions in the two MOFs (see the Supporting Information for $\mathrm{Mg}_{2}$ (dobpdc) data). While the local $\mathrm{Mg}$ environment is similar, $\mathrm{Mg}_{2}$ (dobpdc) has longer organic linkers, allowing us to examine how the linkers and overall framework structure affect the electronic interactions at the binding sites. $\mathrm{Mg}_{2}$ (dobpdc) was originally synthesized to facilitate functionalization with $N, N^{\prime}$-dimethylethylenediamine (mmen), which further increases $\mathrm{CO}_{2}$ adsorption efficiency. ${ }^{19,38}$ We collected $\mathrm{Mg}$ Kedge spectra of the functionalized form mmen- $\mathrm{Mg}_{2}$ (dobpdc) to compare the electronic interactions of $\mathrm{CO}_{2}$ and mmen with the $\mathrm{Mg}$ sites in this MOF. Like DMF, mmen interacts strongly with the $\mathrm{Mg}$ site and provides a useful contrast to the weaker interaction with $\mathrm{CO}_{2}$. Finally, we collected the NEXAFS spectrum of cubic $\mathrm{MgO}$ for energy calibration and for assistance in the interpretation of observed spectral changes.

A representative series of spectra for one of the Mg-MOF-74 samples is shown in Figure 1a. A distinct pre-edge peak is observed for the activated sample that is suppressed and blueshifted when $\mathrm{CO}_{2}$ gas is introduced, accompanied by an increase in intensity and slight blue shift of the strongest main edge feature. These features evolve progressively as a function of gas pressure. The nonzero experimental pressures span 


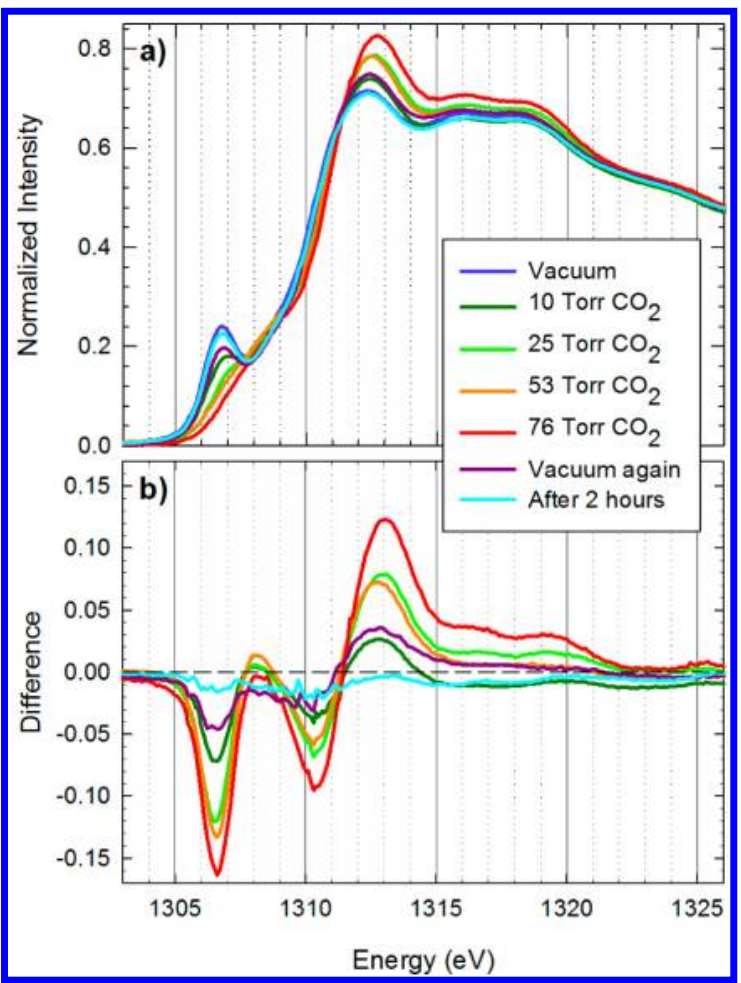

Figure 1. (a) Mg K-edge spectra of Mg-MOF-74 under vacuum, with increasing $\mathrm{CO}_{2}$ gas pressures, under vacuum again after pumping the gas out, and a final spectrum collected $2 \mathrm{~h}$ later. The spectra taken with gas correspond to $\sim 0.28, \sim 0.46, \sim 0.57$ and $\sim 0.65 \mathrm{CO}_{2}$ molecules per $\mathrm{Mg}$ site, in order of increasing pressure. All spectra were collected at an ambient temperature of $295 \mathrm{~K}$. (b) Difference spectra generated by subtracting the initial vacuum spectrum from all subsequent spectra.

$\sim 0.28$ to $\sim 0.65 \mathrm{CO}_{2}$ molecules per $\mathrm{Mg}$ site, according to adsorption isotherm data. ${ }^{1}$ When the gas is pumped out, the spectral changes reverse, with the pre-edge feature reappearing within minutes and nearly full reversion to the original vacuum spectrum after $2 \mathrm{~h}$. Figure $1 \mathrm{~b}$ shows the difference spectra generated by subtracting the initial vacuum spectrum from each subsequent spectrum. The two negative regions at lower energy correspond to the suppression of the pre-edge peak and blue shift of the main edge absorption onset upon gas adsorption, while the broad positive region at higher energy corresponds to the increased intensity in the main edge features upon adsorption. The integral under some of the difference curves in Figure $1 \mathrm{~b}$ is effectively 0 , consistent with the dipole oscillator sum rule prediction that observed spectral changes result from redistribution of $\mathrm{Mg} 3 \mathrm{p}$ spectral weight as the $\mathrm{Mg}$ environment changes, as discussed below. Since only $\sim 65 \%$ of the $\mathrm{Mg}$ sites are occupied at 76 Torr, the spectrum of the fully saturated MOF can be approximated by subtracting a percentage of the vacuum spectrum from the 76 Torr spectrum, as shown in Figure 2. The result clearly lacks the pre-edge peak that appears at $1306.6 \mathrm{eV}$ for the activated MOF.

Similar spectral changes are observed when other molecules are adsorbed in the MOF. Figure 3a shows the Mg K-edge spectra for activated Mg-MOF-74, Mg-MOF-74 in 76 Torr $\mathrm{CO}_{2}$, and DMF-ligated Mg-MOF-74. Difference spectra are shown in Figure $3 \mathrm{~b}$. In the DMF case we expect the $\mathrm{Mg}$ sites to be fully saturated. The pre-edge shows a very broad, weak shoulder that extends to lower energy, but the changes in the main edge absorption onset and main edge intensity are very

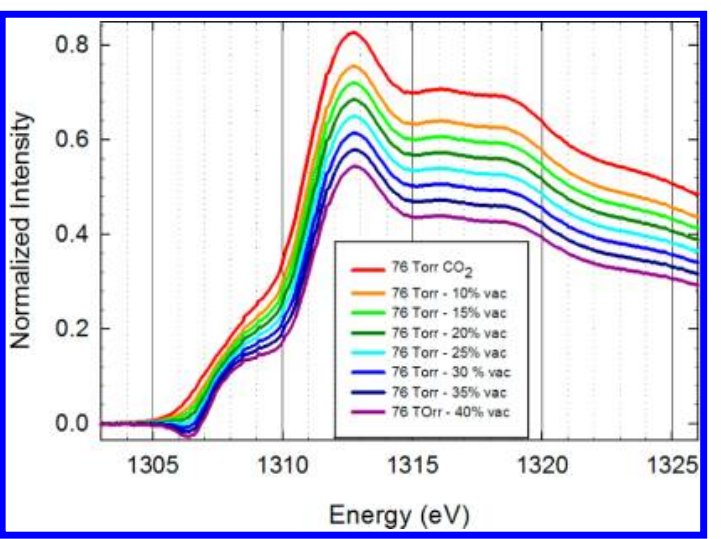

Figure 2. Mg K-edge NEXAFS spectrum of Mg-MOF-74 in 76 Torr $\mathrm{CO}_{2}$ gas and the same spectrum with various percentages of the vacuum spectrum subtracted. These spectra therefore approximate the shape of the spectrum of Mg-MOF-74 on saturatation with $\mathrm{CO}_{2}$ gas. Note the absence of the prepeak at $1306.6 \mathrm{eV}$ that is characteristic of the empty $\mathrm{Mg}$ binding sites.

similar to those of the $\mathrm{CO}_{2}$ case. The pre-edge peak is absent when $\mathrm{CO}_{2}$ or DMF is present, indicating that it is associated with the coordinatively unsaturated $\mathrm{Mg}$ sites in the activated MOF. Similar pre-edge features have been observed for other metal K-edges of crystalline solids and theorized to arise when local symmetry around the metal site is broken by thermal motion, leading to mixing of some metal $\mathrm{p}$ character into the predominantly metal $s$ character of the lowest energy excitations at that site. ${ }^{39-44}$ Core-excited states comprising a 1s hole and local electronic s character at the same site are inaccessible in NEXAFS due to the dipole selection rule. Valid for optical excitations with low momentum transfer, the selection rule permits electronic transitions with an associated change in angular momentum of \pm 1 . However, the $s-p$ mixing induced by broken symmetry renders these low-energy excited states optically active and evident as pre-edge features in the NEXAFS spectrum. The unique and highly anisotropic squarepyramidal geometry at the $\mathrm{Mg}$ sites in activated Mg-MOF-74 induces similar, but much more pronounced, mixing of $\mathrm{Mg} 3 \mathrm{p}$ and $3 \mathrm{~s}$ character in the low-energy excited states, resulting in strong pre-edge $\mathrm{X}$-ray absorption, as we observe. The suppression of the pre-edge peak upon adsorption of $\mathrm{CO}_{2}$ and DMF can therefore be explained via a partial restoration of octahedral symmetry around the $\mathrm{Mg}$ sites in the MOF.

This interpretation is confirmed using DFT calculations to directly examine the $\mathrm{Mg} 1 \mathrm{~s}$ core-excited states in the MOF and their contributions to the NEXAFS spectrum. Calculated NEXAFS spectra for the three experimental cases for $\mathrm{Mg}$ MOF-74 are shown in Figure 3c, along with the transition probabilities from which the spectra were generated. Difference spectra are shown in Figure 3d. The intensity of a given transition is dependent not only on the overlap between the empty final excited state and the Mg 1s orbital but also on the degree of $\mathrm{p}$ character of the final state within this overlap. Final states that are highly delocalized or have low $\mathrm{p}$ character at the $\mathrm{Mg}$ site will have weak transition probabilities.

We find generally good agreement between experimental and calculated spectra. Spectral features are reproduced at the correct energies, and the spectral changes upon gas adsorption-including the suppression of the pre-edge peak, blue shift of the main edge onset, and increased intensity and blue shift of the main edge features-are reproduced as well. 

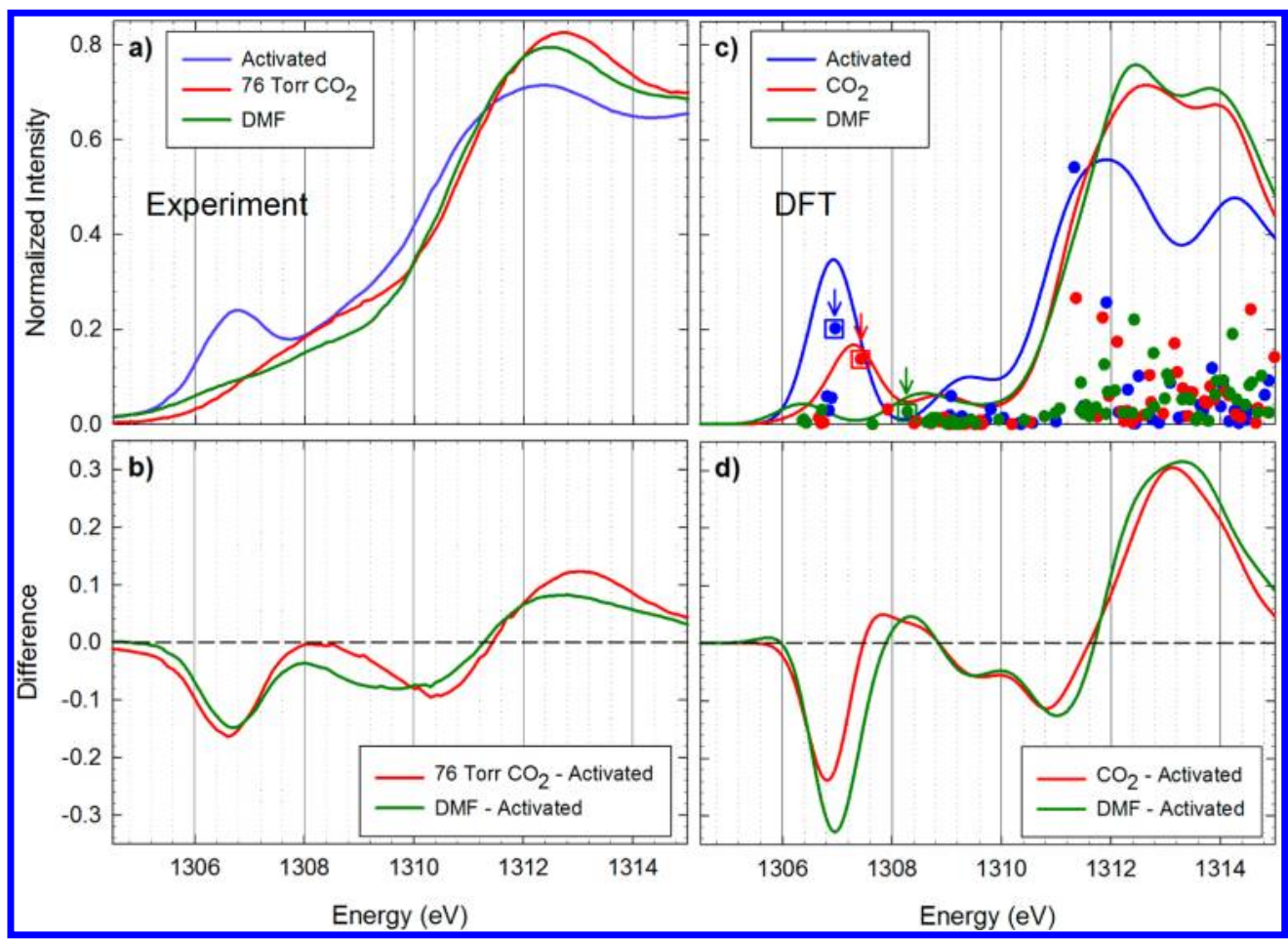

Figure 3. (a) NEXAFS spectra for activated Mg-MOF-74 (blue), Mg-MOF-74 with 76 Torr $\mathrm{CO}_{2}$ (red), and Mg-MOF-74 with DMF solvent (green). (b) Difference spectra generated by subtracting the activated MOF spectrum from the 76 Torr $\mathrm{CO}_{2}$ (red) and DMF (green) spectra. (c) Calculated NEXAFS spectra (lines) for activated Mg-MOF-74 (blue), Mg-MOF-74 with adsorbed $\mathrm{CO}_{2}$ (red), and DMF ligated Mg-MOF-74 (green), along with the transition probabilities (circles) used to generate the spectra, corresponding to individual excited states. Only the transitions to states at the Brillouin zone center $(k=0)$ are shown. The intensity of the pre-edge features is dominated by a single transition in each case, marked with a colored square and arrow. (d) Difference spectra generated by subtracting the computed activated MOF spectrum from the computed adsorbed $\mathrm{CO}_{2}$ (red) and computed DMF ligated (green) spectra.

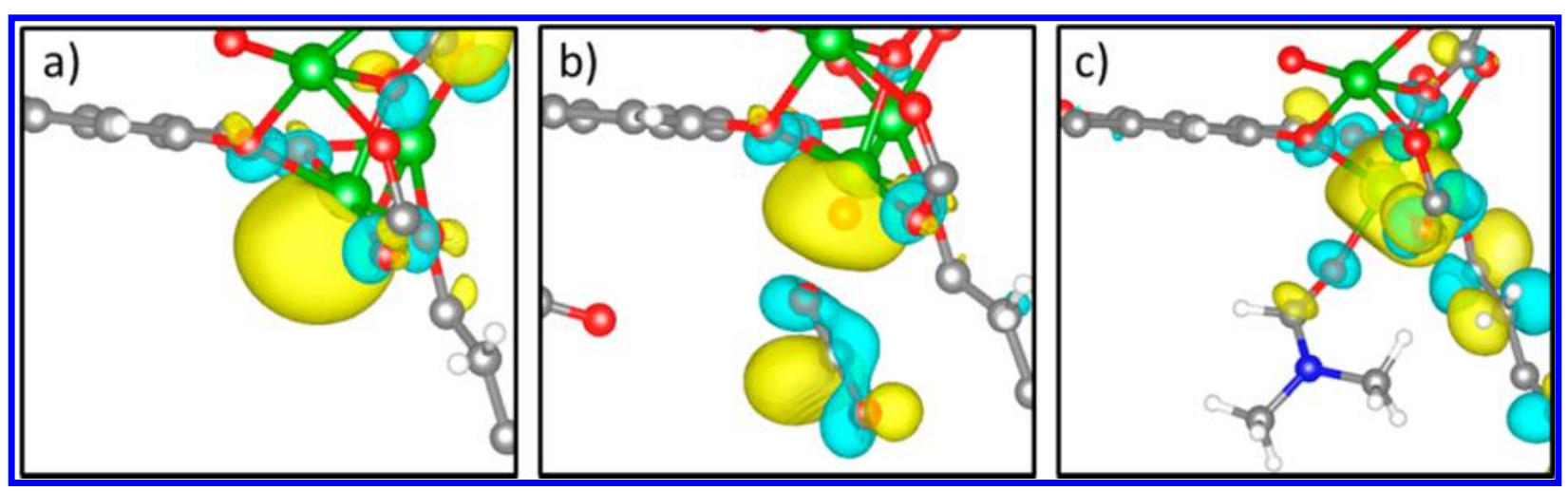

Figure 4. (a) Excited state wave function corresponding to the blue marked transition in Figure 3c. Magnesium atoms are shown in green, oxygen atoms in red, carbon atoms in gray, and hydrogen atoms in white. The two phases of the wave function are shown in yellow and teal. (b) Excited state wave function corresponding to the red marked transition in Figure 3c. (c) Excited state wave function corresponding to the green marked transition in Figure 3c. While these electronic states extend to neighboring linker and ligand molecules, the spectral features given by the calculation sample these states only near the $\mathrm{Mg}$ nucleus.

Minor discrepancies likely arise from the effects of thermal motion in the ambient-temperature experimental spectra; computed spectra use $0 \mathrm{~K}$ structures. For example, the preedge peak is much more intense in the computed spectra, especially when $\mathrm{CO}_{2}$ is adsorbed. Previous studies of condensed-phase systems have shown that the lower-energy spectral features are more sensitive to thermal broadening; ${ }^{45}$ these low-energy states are highly localized and resemble molecular states with appropriately high sensitivity to atomic positions, whereas higher-energy features resemble bands that are less sensitive to these positions. The combination of $\mathrm{CO}_{2}$ adsorption and thermal broadening in Mg-MOF-74 could therefore reduce the intensity of the pre-edge peak until it is no longer observable.

The importance of dynamics for adsorbed $\mathrm{CO}_{2}$ in Mg-MOF74 at ambient temperature has been demonstrated previously, ${ }^{15}$ so it is possible that the thermal motion of the adsorbed $\mathrm{CO}_{2}$ itself affects the spectrum. To approximate this, we applied a series of translations and rotations to the adsorbed $\mathrm{CO}_{2}$ and computed the spectral sensitivity to these motions (see Figure S4 for details). Clear changes in both the pre-edge and main edge regions of the spectrum appear, but the Boltzmann- 
weighted average yields a spectrum that is indistinguishable from the zero-Kelvin spectrum. This suggests that the NEXAFS spectrum is primarily sensitive to thermal motion of the framework itself rather than of the adsorbed species.

2. X-ray Excited-State Analysis. Given the qualitative agreement between experiment and theory, a closer examination of the excited electronic states associated with the computed transitions provides further insight into the origin of the pre-edge peak. For activated Mg-MOF-74, the intensity in the pre-edge region arises primarily from a single transition, marked in Figure 3c and with the corresponding excited state wave function shown in Figure 4a. This excited state has a clear contribution from the $\mathrm{Mg} 3 \mathrm{~s}$ orbital and, as such, should be inaccessible in NEXAFS. In the activated MOF, the state extends into the unoccupied apical site and is no longer centered on the $\mathrm{Mg}$ nucleus, which implies a nonzero first moment with respect to the $\mathrm{Mg}$ 1s core orbital and hence an allowed optical transition. The other empty states in the preedge region also feature this localized, distorted lobe at the $\mathrm{Mg}$ site but have stronger hybridization with the $\pi$ systems on the ligands, delocalizing the states, which reduces overlap with the core orbital and lowers transition intensities. When $\mathrm{CO}_{2}$ is adsorbed in the MOF, the pre-edge feature is once again dominated by a single transition. The corresponding final state wave function is shown in Figure $4 \mathrm{~b}$. It looks similar to the most intense state in the activated MOF, again showing a localized, distorted lobe at the $\mathrm{Mg}$ site, but now there is clear hybridization between this lobe and the $\pi^{*}$ orbital of the adsorbed $\mathrm{CO}_{2}$ molecule. While the adsorbed $\mathrm{CO}_{2}$ does not appreciably restore spherical symmetry at the $\mathrm{Mg}$ site, the intensity of the transition is reduced because the hybridization with the $\mathrm{CO}_{2}$ molecule delocalizes the state.

When DMF coordinates to the Mg site, the two low-intensity features in the pre-edge region each have a dominant transition. The low-energy transition corresponds to a state consisting primarily of the $\pi^{*}$ orbital of the DMF molecule, showing very slight hybridization with the distorted lobe at the $\mathrm{Mg}$ site. The high-energy transition, marked in Figure $3 c$ and with the corresponding wave function shown in Figure 4c, shows a less distorted lobe that is now centered on the $\mathrm{Mg}$. The DMF molecule partially restores the octahedral symmetry around the $\mathrm{Mg}$ and the spherical symmetry of the empty electronic state, reducing the intensity of the NEXAFS transition. The dipole moment and larger polarizability of DMF with respect to $\mathrm{CO}_{2}$ result in a larger binding energy and shorter bond length, as reported in the Experimental Section. This indicates that strongly interacting species will have more pronounced spectral signatures upon adsorption at open metal sites. While adsorbed $\mathrm{CO}_{2}$ and DMF are distinct in the computed spectra, they are more difficult to distinguish experimentally, implying that thermal motion may mask some of the spectral signatures for different adsorbed species.

Measurements of $\mathrm{Mg}_{2}$ (dobpdc) show similar, but less pronounced, pre-edge behavior (see the Supporting Information for details). This indicates that the pre-edge feature is a general phenomenon that will appear whenever there is a change in local symmetry at $\mathrm{Mg}$ sites in a material. To test this, we computed the NEXAFS spectrum of octahedral $\mathrm{MgO}$ as well as two distorted cases, in which the bond length of the apical oxygen was increased by 0.5 and $1.0 \AA$. The results are shown in Figure 5. For octahedral $\mathrm{MgO}$, some low-energy transitions have little or no intensity in the spectrum, consistent with previous studies of $\mathrm{MgO}^{26,46}$ The final state wave

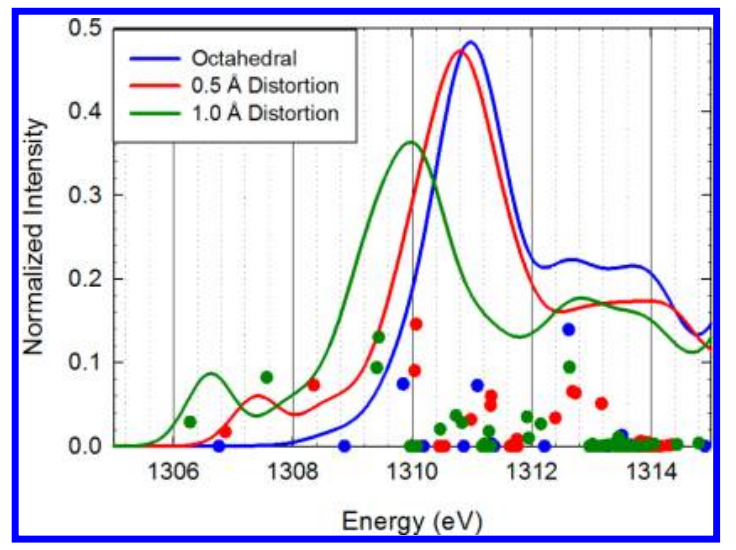

Figure 5. Calculated NEXAFS spectra for octahedral $\mathrm{MgO}, \mathrm{MgO}$ in which the $\mathrm{Mg}-\mathrm{O}$ bond distance of the apical oxygen has been increased by $0.5 \AA$, and $\mathrm{MgO}$ in which the $\mathrm{Mg}-\mathrm{O}$ bond distance of the apical oxygen has been increased by $1.0 \AA$. Also shown are the transition probabilities corresponding to $\gamma$ point states, which form the lower bound of any spectral feature in the simulated spectrum. Note that the low-energy transitions for octahedral $\mathrm{MgO}$ have zero intensity due to the octahedral symmetry. With distortions to the apical oxygen, these transitions become active in the spectrum, creating a new preedge peak.

functions for these transitions resemble the case of DMFligated Mg-MOF-74 in Figure 4c, consisting primarily of the empty Mg 3s orbital centered on the Mg site (see Figure S5 in the Supporting Information for the $\mathrm{MgO}$ wave functions). When the apical oxygen bond length is increased, spectral features similar to those observed for Mg-MOF-74 appear. In particular, we find a distinct new pre-edge feature which arises from an asymmetric, distorted electronic state around the $\mathrm{Mg}$ that resembles that seen for activated Mg-MOF-74 (Figure 4a). Further increasing the $\mathrm{MgO}$ bond length creates a more dramatic modification of the pre-edge. This confirms that breaking the octahedral symmetry at the $\mathrm{Mg}$ site creates the spectral features observed for Mg-MOF-74, and the spectral sensitivity to the $\mathrm{Mg}-\mathrm{O}$ bond length in $\mathrm{MgO}$ underscores the importance of framework dynamics in spectral modeling.

\section{CONCLUSION}

We find strong sensitivity of the Mg K-edge NEXAFS spectrum to the adsorption of $\mathrm{CO}_{2}$ gas and DMF in Mg-MOF-74 and to adsorption of $\mathrm{CO}_{2}$ gas and mmen in $\mathrm{Mg}_{2}$ (dobpdc). The open coordination at the $\mathrm{Mg}$ sites in these materials activates otherwise forbidden transitions in the NEXAFS spectrum, creating pre-edge features that are signatures of the open metal sites. While all of the adsorbed species reduce the intensity of these pre-edge features, we find that more strongly adsorbed molecules result in a more octahedral-like geometry around the $\mathrm{Mg}$ and a stronger spectral signature. This detailed and direct information about the electronic structure and interactions at the active $\mathrm{Mg}$ sites fills a critical gap in validating empirical, experimental, and theoretical models for the fundamental gas interaction mechanisms in these materials. We find good agreement between our ambient-temperature measurements and DFT calculations based on static structures, with minor discrepancies that are likely due to vibrational motion in the framework which instantaneously modifies the symmetry at the $\mathrm{Mg}$ sites.

The high spectral sensitivity to the $\mathrm{Mg}$ site symmetry underscores the versatility of NEXAFS for in situ gas 
adsorption measurements in nanoporous materials such as MOFs, especially those with chemically distinct open metal sites. NEXAFS can also probe adsorption occurring at the organic linkers or functional additives in MOFs, to explore more complex adsorption interactions. This approach provides a new method to monitor the chemistry within working MOFs with chemical specificity that will be useful to test theoretical models for structure, chemistry, and potentially dynamics and kinetics. Its systematic application may inform the design of MOFs with improved performance in specific tasks.

\section{ASSOCIATED CONTENT}

\section{S Supporting Information}

Text and figures giving synthetic details and sample preparation, adsorption isotherms, NEXAFS spectra and DFT calculations of $\mathrm{MgO}$ with and without apical distortion, NEXAFS spectra and DFT calculations of $\mathrm{Mg}_{2}$ (dobpdc). This material is available free of charge via the Internet at http://pubs.acs.org.

\section{AUTHOR INFORMATION}

\section{Corresponding Author}

jbkortright@lbl.gov

\section{Author Contributions}

All authors have given approval to the final version of the manuscript.

Notes

The authors declare no competing financial interest.

\section{ACKNOWLEDGMENTS}

This work was supported by the Center for Gas Separations Relevant to Clean Energy Technologies, an Energy Frontier Research Center funded by the U.S. Department of Energy, Office of Science, Office of Basic Energy Sciences, under Award Number DE-SC0001015. Portions of this work were performed as User Projects at the Advanced Light Source and Molecular Foundry, both at Lawrence Berkeley National Laboratory and supported by the Office of Science, Office of Basic Energy Sciences, of the U.S. Department of Energy under Contract No. DE-AC02-05CH11231. Calculations used LBNL Lawrencium, the National Energy Research Scientific Computing Center, LBNL, and the Molecular Foundry computing resources nano and vulcan, managed by the High Performance Computing Services Group, LBNL.

\section{REFERENCES}

(1) Caskey, S. R.; Wong-Foy, A. G.; Matzger, A. J. J. Am. Chem. Soc. 2008, 130, 10870.

(2) Dietzel, P. D. C.; Besikiotis, V.; Blom, R. J. Mater. Chem. 2009, 19, 7362.

(3) Herm, Z. R.; Swisher, J. A.; Smit, B.; Krishna, R.; Long, J. R. J. Am. Chem. Soc. 2011, 133, 5664.

(4) Herm, Z. R.; Krishna, R.; Long, J. R. Microporous Mesoporous Mater. 2012, 151, 481.

(5) Yu, D.; Yazaydin, A. O.; Lane, J. R.; Dietzel, P. D. C.; Snurr, R. Q. Chem. Sci. 2013, 4, 3544.

(6) Britt, D.; Furukawa, H.; Wang, B.; Glover, T. G.; Yaghi, O. M. Proc. Natl. Acad. Sci. U.S.A. 2009, 106, 20637.

(7) Valenzano, L.; Civalleri, B.; Chavan, S.; Palomino, G. T.; Arean, C. O.; Bordiga, S. I. Phys. Chem. C 2010, 114, 11185.

(8) Bao, Z.; Yu, L.; Ren, Q.; Lu, X.; Deng, S. J. Colloid Interface Sci. 2011, 353, 549 .

(9) Mason, J. A.; Sumida, K.; Herm, Z. R.; Krishna, R.; Long, J. R. Energv Environ. Sci. 2011, 4, 3030.
(10) Wong, A.; Ida, R.; Mo, X.; Gan, Z.; Poh, J.; Wu, G. J. Phys. Chem. A 2006, 110, 10084.

(11) Guzei, I. A.; McGaff, R. W.; Kieler, H. M. Acta Crystallogr., Sect. C: Crust. Struct. Commun. 2005, 61, M472.

(12) Dietzel, P. D. C.; Johnsen, R. E.; Fjellvag, H.; Bordiga, S.; Groppo, E.; Chavan, S.; Blom, R. Chem. Commun. 2008, 5125.

(13) Wu, H.; Simmons, J. M.; Srinivas, G.; Zhou, W.; Yildirim, T. J. Phvs. Chem. Lett. 2010, 1, 1946.

(14) Queen, W. L.; Brown, C. M.; Britt, D. K.; Zajdel, P.; Hudson, M. R; Yaghi, O. M. I. Phys. Chem. C 2011, 115, 24915.

(15) Kong, X.; Scott, E.; Ding, W.; Mason, J. A.; Long, J. R.; Reimer, I. A. I. Am. Chem. Soc. 2012, 134, 14341.

(16) Sumida, K.; Brown, C. M.; Herm, Z. R.; Chavan, S.; Bordiga, S.; Long, J. R. Chem. Commun. 2011, 47, 1157.

(17) Valenzano, L.; Civalleri, B.; Sillar, K.; Sauer, J. J. Phys. Chem. C 2011, 115, 21777.

(18) Poloni, R.; Smit, B.; Neaton, J. B. I. Phys. Chem. A 2012, 116, 4957.

(19) McDonald, T. M.; Lee, W. R.; Mason, J. A.; Wiers, B. M.; Hong, C. S.; Long, J. R. I. Am. Chem. Soc. 2012, 134, 7056.

(20) Shih, O.; England, A. H.; Dallinger, G. C.; Smith, J. W.; Duffey, K. C.; Cohen, R. C.; Prendergast, D.; Saykally, R. J. J. Chem. Phys. 2013, 139, 035104.

(21) Zegkinoglou, I.; Ragoussi, M.-E.; Pemmaraju, C. D.; Johnson, P. S.; Pickup, D. F.; Ortega, J. E.; Prendergast, D.; de la Torre, G.; Himpsel, F. J. I. Phvs. Chem. C 2013, 117, 13357.

(22) Jiang, P.; Prendergast, D.; Borondics, F.; Porsgaard, S.; Giovanetti, L.; Pach, E.; Newberg, J.; Bluhm, H.; Besenbacher, F.; Salmeron, M. I. Chem. Phvs. 2013, 138, 024704.

(23) England, A. H.; Duffin, A. M.; Schwartz, C. P.; Uejio, J. S.; Prendergast, D.; Saykally, R. I. Chem. Phys. Lett. 2011, 514, 187.

(24) Schwartz, C. P.; Uejio, J. S.; Duffin, A. M.; England, A. H.; Kelly, D. N.; Prendergast, D.; Saykally, R. J. Proc. Natl. Acad. Sci. U.S.A. 2010, 107,14008

(25) Wyrick, J.; Kim, D.-H.; Sun, D.; Cheng, Z.; Lu, W.; Zhu, Y.; Berland, K.; Kim, Y. S.; Rotenberg, E.; Luo, M.; Hyldgaard, P.; Einstein, T. L.; Bartels, L. Nano Lett. 2011, 11, 2944.

(26) Lindner, T.; Sauer, H.; Engel, W.; Kambe, K. Phys. Rev. B 1986, $33,22$.

(27) Soler, J. M.; Artacho, E.; Gale, J. D.; Garcia, A.; Junquera, J.; Ordejon, P.; Sanchez-Portal, D. I. Phys.: Condens. Matter 2002, 14, 2745.

(28) Roman-Perez, G.; Soler, J. M. Phys. Rev. Lett. 2009, 103, 096103.

(29) Lee, K.; Murray, E. D.; Kong, L.; Lundqvist, B. I.; Langreth, D. C. Phys. Rev. B 2010, 82, 081101.

(30) Troullier, N.; Martins, J. L. Phys. Rev. B 1991, 43, 1993.

(31) Junquera, J.; Paz, O.; Sanchez-Portal, D.; Artacho, E. Phys. Rev. B 2001, 64, 235111.

(32) Moreno, J.; Soler, J. M. Phys. Rev. B 1992, 45, 13891.

(33) Perdew, J. P.; Burke, K.; Ernzerhof, M. Phvs. Rev. Lett. 1996, 77, 3865.

(34) Rohrer, J.; Hyldgaard, P. Phvs. Rev. B 2011, 83, 165423.

(35) Stöhr, J. NEXAFS Spectroscopy; Springer: New York, 1992.

(36) Prendergast, D.; Galli, G. Phvs. Rev. Lett. 2006, 96, 215502.

(37) Prendergast, D.; Louie, S. G. Phvs. Rev. B 2009, 80, 235126.

(38) Planas, N.; Dzubak, A. L.; Poloni, R.; Lin, L.-C.; McManus, A.; McDonald, T. M.; Neaton, J. B.; Long, J. R.; Smit, B.; Gagliardi, L. J. Am. Chem. Soc. 2013, 135, 7402.

(39) Pascal, T. A.; Boesenberg, U.; Richardson, T. J.; Weng, T.-C.; Sokaras, D.; Nordlund, D.; McDermott, E.; Moewes, A.; Cabana, J.; Prendergast, D. G. J. Chem. Phys. 2013, accepted for publication.

(40) Manuel, D.; Cabaret, D.; Brouder, C.; Sainctavit, P.; Bordage, A.; Trcera, N. Phvs. Rev. B 2012, 85, 224108.

(41) Pantelides, S. T. Phvs. Rev. B 1975, 11, 2391.

(42) Kunz, A. B. Phys. Rev. B 1975, 12, 5890.

(43) Pantelides, S. T.; Brown, F. C. Phvs. Rev. Lett. 1974, 33, 298.

(44) Kunz, A. B.; Collins, T. C.; Devreese, J. T. J. Phys. Part C: Solid State Phys. 1972, 5, 3259. 
(45) Schwartz, C. P.; Uejio, J. S.; Duffin, A. M.; England, A. H.; Prendergast, D.; Saykally, R. J. I. Chem. Phys. 2009, 131, 114509.

(46) Mizoguchi, T.; Tanaka, I.; Yoshiya, M.; Oba, F.; Ogasawara, K.; Adachi, H. Phys. Rev. B 2000, 61, 2180. 